\title{
A Fatal Case of Accidental High-Voltage Electrocution
}

Samadhi H Dandeniya Arachchi ${ }^{1 *} \otimes\left(D\right.$, Rohan P Ruwanpura ${ }^{1}$

${ }^{1}$ Office of the Judicial Medical Officer, Teaching Hospital, Karapitiya, Galle, Sri Lanka.

High voltage electrical injuries are relatively uncommon but exhibit a high rate of mortality. A body of a 39-yearold electrician who had died while working on high tension cables was brought for post-mortem examination. The autopsy revealed first and second-degree burns, exfoliated skin and crocodile flash burns around the neck. There was a penetrating skull fracture with exposed brain and surrounding charring. Microscopic examination revealed evidence of electrocution in the skin and myocardium. Cause of death was stated as multiple injuries following high voltage electrocution. Detailed history, scene visit and proper postmortem examination with histological and toxicological analysis are recommended prior to concluding the cause of death as electrocution

Keywords: High voltage electrocution, histopathology, injury pattern.

Copyright: @ 2018 with the Medico-legal Journal of Sri Lanka.

This is an open-access article distributed under the terms of the Creative Commons Attribution 4.0 International License, which permits unrestricted use, distribution and reproduction in any medium provided the original author and source are credited.

Funding: None,

Competing interests: None

Received: 18 Sep 2018 Accepted revised version: 15 December 2018 Published: 30 December 2018

*Corresponding author: Samadhi DAH, E-mail: dahsamadhid@gmail.com $\bigotimes$, (D) https://orcid.org/0000-0003-4814-6255

Cite this article as: Samadhi DAH, Ruwanpura PR. A Fatal Case of Accidental High-Voltage Electrocution. Medico-Legal Journal of Sri Lanka, 2018;6(2):78-83.

DOI: http://dx.doi.org/10.4038/mljsl.v6i2.7379

\section{Introduction}

Electric injuries, a different type of mechanical trauma with a unique injury pattern occur due to lightning, low voltage (less than $600 \mathrm{~V}$ ) or high voltage (more than $600 \mathrm{~V}$ ) electrocution and most of the incidents are accidental and preventable. ${ }^{[1]}$ Though the word "electrocution" has derived from 'electro' and 'execution' it is usually used to address the accidental and suicidal circumstances as well. ${ }^{[2]}$ High voltage electrical injuries are relatively uncommon but, considerably contribute to occupational fatalities with the existence of hazardous exposure during their tasks at workplaces ${ }^{[3]}$. They exhibit a high rate of mortality, and severe systemic complications those who are survived.

This case report discusses the injuries sustained by an adult male following an accidental contact of his head and neck with a high tension wire.

\section{Case Report}

A body of a 39-year-old male, electrician of power supply line maintenance service was brought for the postmortem examination to the mortuary of a tertiary care hospital. According to the investigating police officer that the victim was attending to repair work on a high tension cable post near the city. He was not wearing Personal Protective Equipment (PPE), other than the antiskid belt. The electricity line was switched on while the repair was in progress due to miscommunication, then disconnected soon when short-circuit has been noticed. The victim had suffered high tension shock and died at the scene, but remained attached to the post through supporting protective belt (Fig. 01). 


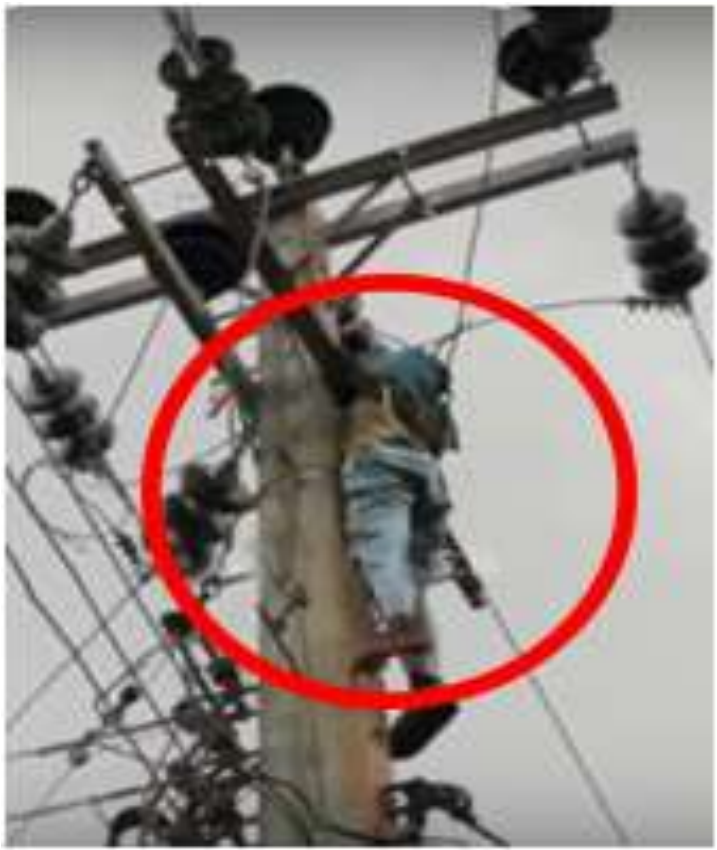

Figure 01: Position of the body at the scene showed direct contact of the head, neck and left shoulder with the electric line.

The autopsy revealed that the deceased was 68 inches $(174 \mathrm{~cm})$ in length, weighing $65 \mathrm{~kg}$, average built and well-nourished male. Protection belts were clipped around the hip and chest, while underlying apparels were ignited and soaked with blood at several areas (Fig. 02). Rigor mortis was fully established and the postmortem lividity was fixed and confined mainly on lower extremities and back aspect of the body.

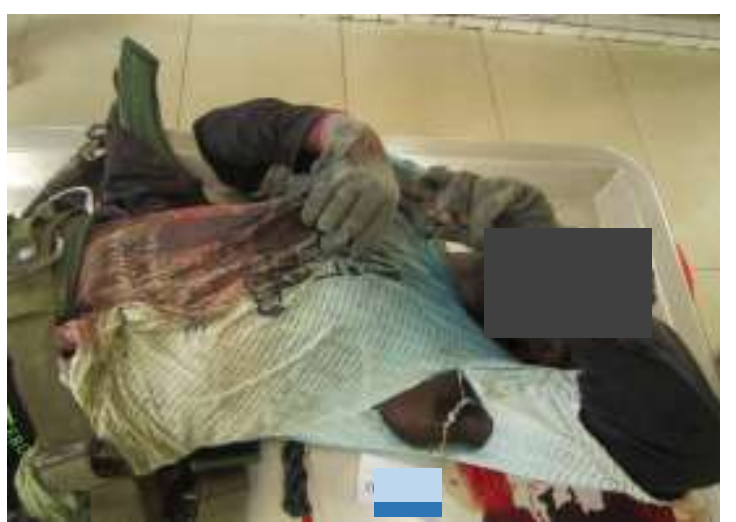

Figure 02: Body with protection belt and burnt clothing

External injuries of multiple first and second degree burn with exfoliated skin were seen on the back of the body (Fig. 03). Electrical burn marks were seen on the limbs (Fig. 04) and soles (Fig. 05).

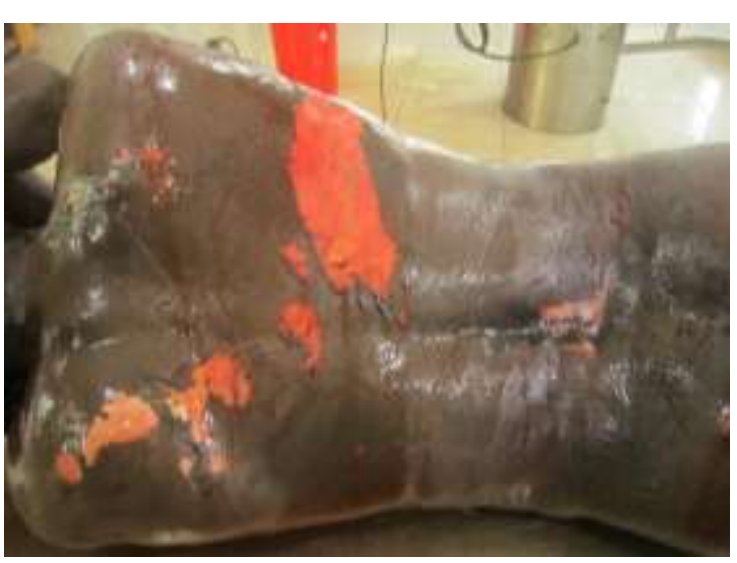

Figure 03: Burns with exfoliation of the skin

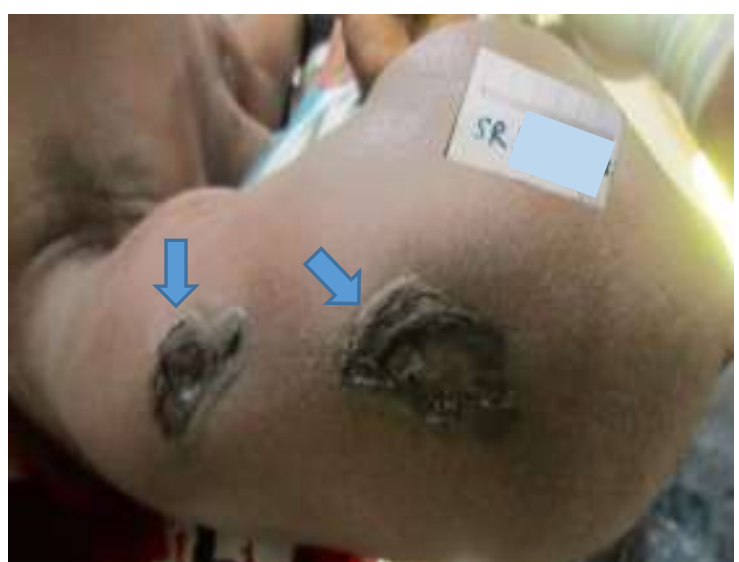

Figure 4: Two Electric burn marks on the upper arm showed by arrows

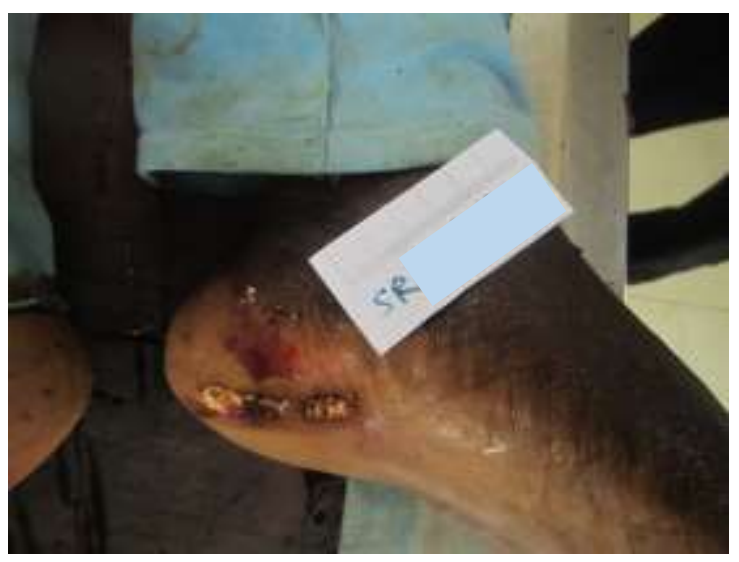

Figure 05: An elongated burn mark on the sole

Multiple punched out lesions (crocodile flash burns) were noted over the nape of the neck (Fig. 06). A penetrating fracture of the skull of about $7 \mathrm{~cm} \times 5 \mathrm{~cm}$ due to the burning of the scalp and the underlying occipital bone was present and the brain was exposed through the charred wound (Fig. 07). Hair around the wound and eyebrows were singed. Internal organs were congested and the heart was cyanotic and rigid. The brain was mildly edematous with congested vessels. 


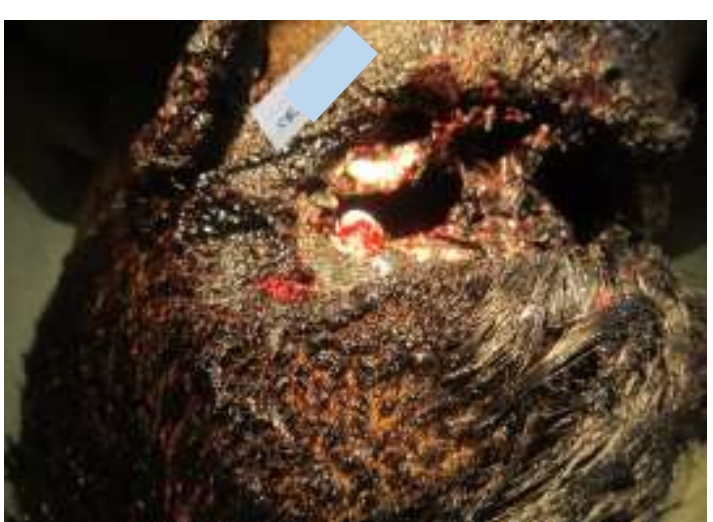

Figure 06: Crocodile flash burns on the nape of the neck

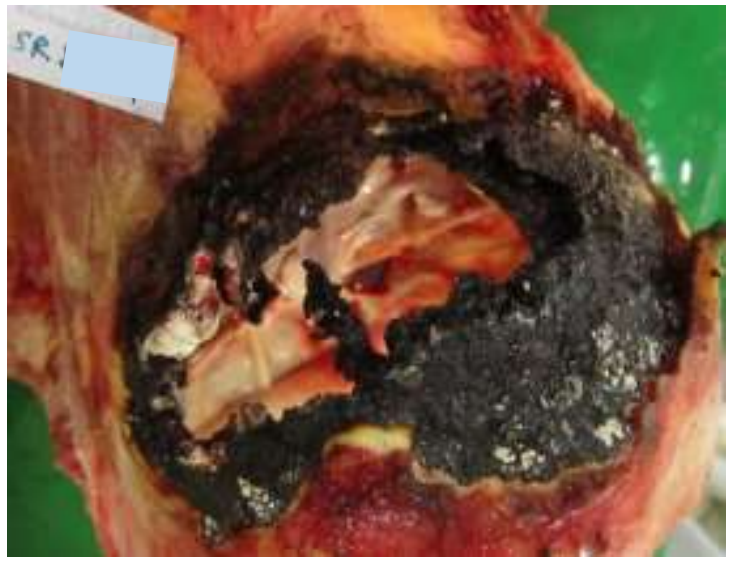

Figure 07: Penetrating deficiency of the skull

Microscopic examination of the burnt skin showed sub-epidermal separation, coagulative necrosis of epidermis (Fig. 08)with nuclear palisading appearance (Fig. 09), distortion of architectural arrangement of hepatocytes (Fig. 10), and while embedded foreign bodies probably originated from metallic components were revealed under polarizing light (Fig. 11).

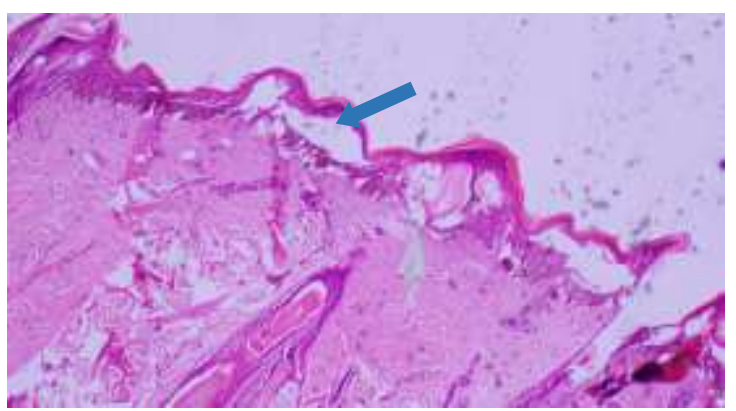

Figure 08: Microphotograph of epidermal separation with micro blisters indicated by an arrow. H\&E x 100

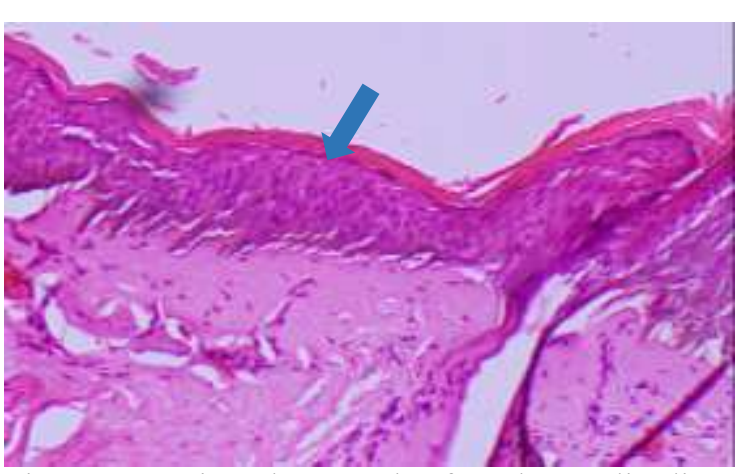

Figure 09: Microphotograph of nuclear palisading indicated by an arrow. H\&E x 100

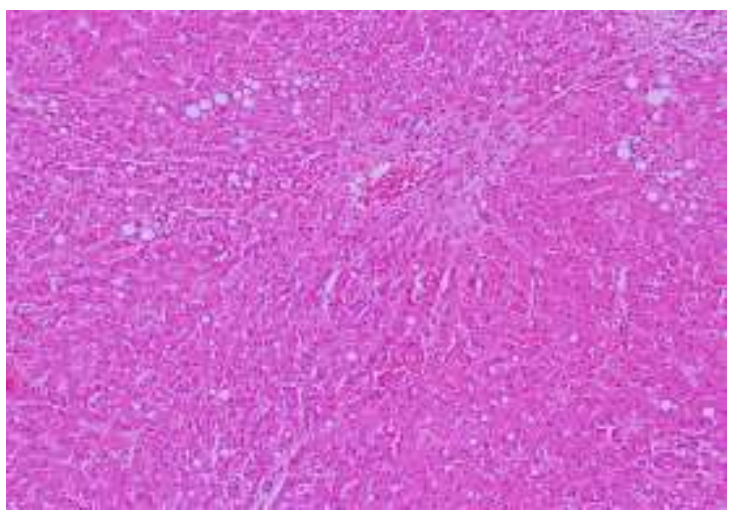

Figure 10 Microphotograph of distortion of liver architecture H\&E x 40

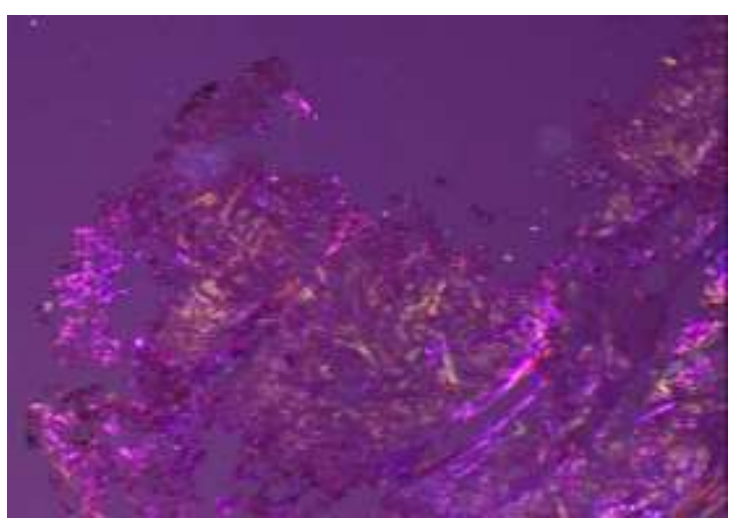

Figure 11: Microphotograph of foreign bodies under polarizing light $\mathrm{x} 100$

The sections of the heart showed myofibril necrosis with square expression of nuclei (green arrow in Fig. 12) and alternative appearance of a bundle of hyper contracted-hyper distended myocytes were the predominant features (Fig. 13). 


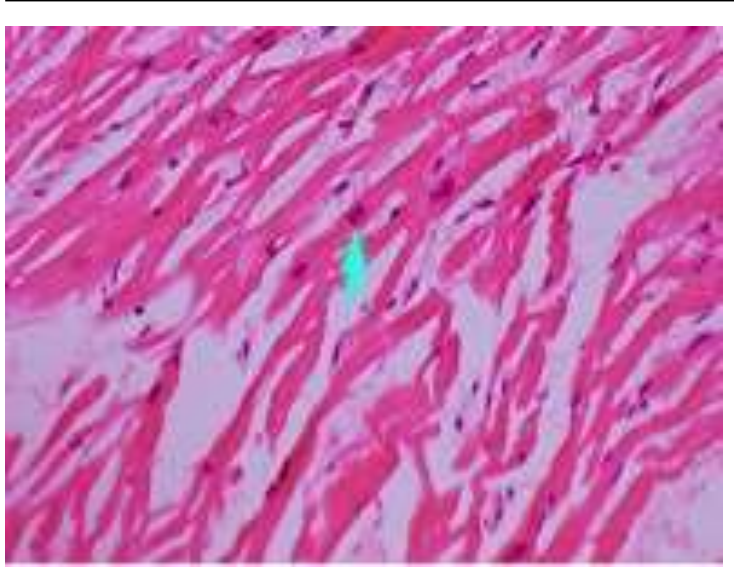

Figure 12: Microphotograph of square-shaped nuclei with necrosis of myocytes indicated by arrow H\&E x 100

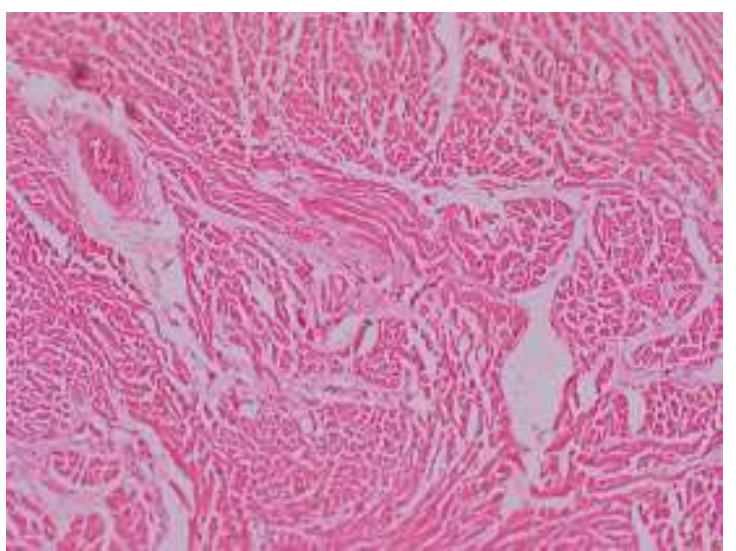

Figure 13: Microphotograph of hyper-contracted bundles of myofibrils H\&E x 40

Massive alveolar destruction is seen in lung histology (Fig. 14). Liver and brain sections showed congestion with some architectural disturbance in histology.

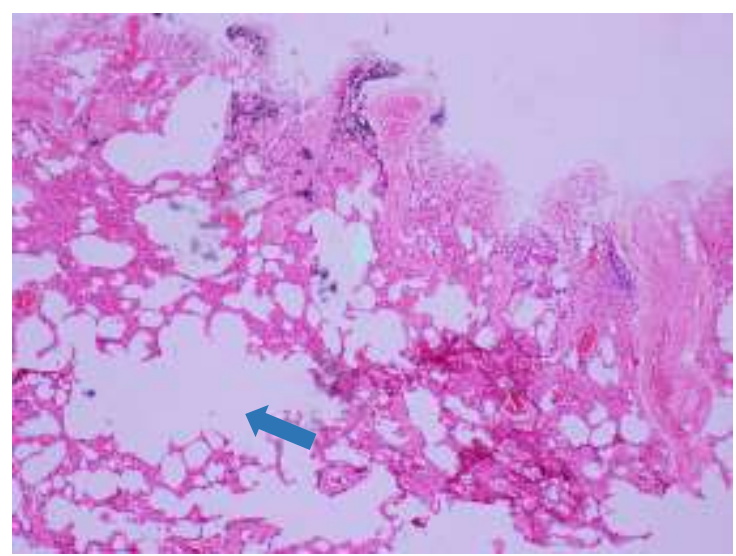

Figure 14: Microphotograph of the destruction of alveoli indicated by an arrow. H\&E x 40

Toxicological studies were not performed as all circumstantial evidence and autopsy features were exclusive of intoxication. However further inquiries revealed that the electric switching on while on repair was due to miscommunication in between supervising officers, which may amount to professional negligence. Though I couldn't follow up the impact with legal affairs and its further involvement the dependents of the deceased should be liable to workman's compensation as the death is solely an accidental in nature and occurred during his working time. The cause of death was given as multiple injuries following High Voltage Electrocution.

\section{Discussion}

Though the incidence of it is considerably low compared to the other types of electrical injuries, the fatality rate is very high with direct contact or indirect arcing or flashover effect. Among these fatalities reported, most of the victims are manual workers [3] and it emphasizes the presence of electricity in most of the worksites and exposure while performing their tasks.

According to the death investigation of Sri Lanka, such deaths are required to be an inquest and it further executes fair justice to employee and employer. Furthermore, it helps for subsequent compensation, claiming insurance etc. ${ }^{[4]}$

Diagnosis of high voltage electrocution is sometimes difficult in the absence of history and circumstantial evidence, as the pathognomic features like electric marks and joule burns are often seen only with low or medium voltage current involvement and also both entry and exit marks are seen together only in $20 \%$ cases. $^{[5]}$ Proper differentiation of the injuries and histopathological changes of electrocution are more valuable in those cases.

The present case report refers to an electrician who was accidentally electrocuted with a high tension wire discusses the injury pattern, gross and the histopathological consequences of tissues following high voltage electrocution.

Electrical burns occur due to the conversion of electric current to heat within the body, and this can be either entry or exit marks. According to the circumstantial evidence, the diseased had been contacted with the current wire that carries about $33000 \mathrm{~V}$ of high voltage for few minutes as the protective belts worn by the deceased has prevented him from thrown away effect. The prolong contact has lead the electric mark at the contacted site (entry mark) to be unrecognizable and the area around the nape of the neck was charred with burning of the scalp and the skull bone in the occipital region. Multiple electrical marks were identified during the autopsy of the victim, and they were confined to the upper limbs and sole. Those marks showed the characteristic appearance of dry, firm to hard craters with ragged edges. 
Another type of electric mark is a spark burn which occurs with the presence of a gap between the skin and the conductor. In high voltage electrocution, multiple spark burns crack into the victim causes a large area of tissue damage and give rise to 'crocodile skin' appearance, here it was seen around the contacted site; the nape of the neck. There were $1^{\text {st }}$ and $2^{\text {nd }}$ degree burns also with flash effect over the back of the body and also singeing of hair and eyelashes due to the short range flashes.

Microscopic examination of skin around the burnt area was done with normal Haematoxylin \& Eosin (H\&E) staining and showed characteristic subepidermal separation, nuclear streaming and Separation of the epidermis is due to micro blisters that formed during the cooking effect of tissues with the strong heat and the large vacuoles also identified within the epidermal layer. High-temperature burns denature the collagen materials in the skin which then responsible for the bluish staining of it in $\mathrm{H} \& \mathrm{E}$ staining. Elongated and tightly packed nucleus in the basal layer of epidermis causes streaming of the nuclei which give rise to nuclear palisading effect and by far it is the most characteristic histological feature seen in electrical burns and seen in the majority of cases ${ }^{[6,7]}$ But these nuclear changes are not specific for electrocution as it may also find in cauterization, freezing, blunts injuries and blisters formation following barbiturate poisoning. Coagulative necrosis of dermo-epidermal cells presents in $60 \%$ of electrocuted deaths. ${ }^{[7]}$ Volatilized metal particles from the conductor can also be specifically identified in the contact site histology specimens with polarizing light (Fig. 10).In this study, all the above mentioned histological changes were present in the skin.

Electrical injuries lead to myocardial damage is either due to direct cellular damage or provoking cardiac arrhythmias. Ventricular fibrillation is the most common mechanism of death identified in electrocution. Necrotic and fragmented myocardial cells with round or square shaped nuclei were seen in the microscopic section of the diseased heart, and this with the absence of cellular reaction suggests an instantaneous death. This feature of myofibre breakdown was seen in $90 \%$ of cases in a study conducted by Vittorio Fineschi et al. ${ }^{[8]}$ Intermitted bundles of hyper-contracted myocytes were visible with wavy contraction bands suggestive of contraction band necrosis. ${ }^{[7,8]}$ Interstitial haemorrhages also are seen intermittently in microscopy. The two latter described features are not commonly seen in histology.

Immunohistochemistry can also be used as supportive evidence for microscopic changes which was not used in this case. The immune reaction for
Caspase 3 is important in identifying necrosis and apoptosis. ${ }^{[9]}$

As none of these histological changes is specific for electrocution several features have to be considered with the other evidence. On the other hand, very similar pathological changes can be produced in postmortem electrocution. This will bring the importance of history, circumstantial evidence, injury identification with gross and histological changes taking into consideration in post-mortem investigation prior to concluding the cause of death as electrocution.

The distinguishable finding of this case is a large penetrating skull fracture associated with direct damage of the brain. High-voltage electrocution injuries are a serious problem with potential for both immediate and delayed neurologic sequelae in survivals. ${ }^{[10]}$ It is possible that even if the pathway of electricity does not directly cross the brain, cortisol and the excitatory neurotransmitter glutamate may still be stimulated via the physical and psychological trauma and the "excitatory" impact of the electrical insult.

Complete and properly wearied PPE (including insulated safety gloves, Arc flash clothing, boots, Hearing protective devices, safety belts etc.) Minimize the severity of the injuries if a person accidentally contacts with high tension wires, but in this case, the diseased was only with anti-skid belts and gloves.

\section{Conclusion and Recommendation}

The incidence of high voltage electrocution is somewhat rare than other electrical injuries according to several studies done in several countries. This case warrants the need of wearing personal protective equipment those who engaged in electrical work and the proper inter-personal communication during their tasks. Gross findings of postmortem examination and features in microscopic examination suggest the cause of death as electrocution, but the same changes can be produced by certain types of other injuries and by the artefacts. A detailed history regarding the incident, scene visit and proper postmortem examination with the histological and toxicological analysis are recommended prior to concluding the cause of death as high tension electrocution.

\section{References}

1. Gupta, B. D., R. A. Mehta, and M. M. Trangadia. "Profile of deaths due to electrocution: A retrospective study." J Indian Acad Forensic Med. 2012; 34(1): 13-5. 
2. Wick R, Gilbert JD, Simpson E, Byard RW. Fatal electrocution in adults - a 30-year study. Medicine, science and the law. 2006 Apr;46(2):166-72.

3. Kisner S, Casini V. Epidemiology of electrocution fatalities: 1998. In: Worker deaths by electrocution: A summary of NIOSH Surveillance and Investigative Findings. Washington, DC, Department of Health and Human Services (NIOSH), Publication No. 98131:9-19.

4. Life insurance policy: Is it an indication for inquest? AN Vadysinghe, AMG Abeysekara, MDP Gunasena, RMUC Ratnayake. Sri Lanka Journal of Forensic Medicine, Science and Law. May 2012;3(1): 23-24.

5. Kumar SS, Narayan AR, Gopal S, Kumar JG, Agrawal A. High voltage electrical shock with multiple life-threatening injuries. International journal of critical illness and injury science. 2015 Oct;5(4):266.

6. BadawySM, Mahmoud BL, Hamad SAEH, Fiky AKE, Agmy SESE. Effects of lowvoltage electrocution on hearts of male albino rats: a histopathological and immunohistochemical study. Menoufia Medical Journal. 2015; 28(4):941
7. Vishwakanth B, Shruthi P. Low voltage electrocution deaths and histopathological findings: One-year prospective autopsy study. J Curr Forensic Sci Res. 2015;1(2):1-5

8. Finesch V, Karch SB, D'Errico S, Pomara C, Riezzo I, Turillazzi E et al. "Cardiac pathology in death from electrocution." International journal of legal medicine. 2006;120(2): 79-82

9. Kandeel S, Elhosary NM, El-Noor MM, Balaha M. Electric injury-induced Purkinje cell apoptosis in rat cerebellum: Histological and immunohistochemical study. Journal of chemical neuroanatomy. 2017 Apr 1;81:87-96. Doi: 10.1016/j.jchemneu.2017.02.010.

10. Bailey B, Gaudreault P, Thivierge RL. Neurologic and neuropsychological symptoms during the first year after an electric shock: Results of a prospective multicenter study. Am J Emerg Med. 2008;26(4):413-8 